\title{
Article \\ Vibrational Excitation Cross-Section by Positron Impact: A Wave-Packet Dynamics Study
}

\author{
Luis A. Poveda ${ }^{1, * \mathbb{D}}$, Marcio T. do N. Varella ${ }^{2} \mathbb{D}$ and José R. Mohallem ${ }^{3} \mathbb{D}$ \\ 1 Departamento de Física, Centro Federal de Educação Tecnológica de Minas Gerais, \\ Belo Horizonte 30421-169, MG, Brazil \\ 2 Intituto de Fisica, Universidade de São Paulo, São Paulo 05315-970, SP, Brazil; mvarella@if.usp.br \\ 3 Departamento de Física, Universidade Federal de Minas Gerais, Belo Horizonte 30123-970, MG, Brazil; \\ rachid@fisica.ufmg.br \\ * Correspondence: poveda@cefetmg.br
}

check for updates

Citation: Poveda, L.A.; Varella, M.T.d.N.; Mohallem, J.R. Vibrational Excitation Cross-Section by Positron Impact: A Wave-Packet Dynamics Study. Atoms 2021, 9, 64. https:// doi.org/10.3390/atoms9030064

Academic Editor:

Grzegorz Piotr Karwasz

Received: 17 August 2021

Accepted: 7 September 2021

Published: 9 September 2021

Publisher's Note: MDPI stays neutral with regard to jurisdictional claims in published maps and institutional affiliations.

Copyright: (c) 2021 by the authors. Licensee MDPI, Basel, Switzerland. This article is an open access article distributed under the terms and conditions of the Creative Commons Attribution (CC BY) license (https:// creativecommons.org/licenses/by/ $4.0 /)$.

\begin{abstract}
The vibrational excitation cross-section of a diatomic molecule by positron impact is obtained using wave-packet propagation techniques. The dynamics study was carried on a twodimensional potential energy surface, which couples a hydrogenlike harmonic oscillator to a positron via a spherically symmetric correlation polarization potential. The cross-section for the excitation of the first vibrational mode is in good agreement with previous reports. Our model suggests that a positron couples to the target vibration by responding instantly to an interaction potential, which depends on the target vibrational coordinate.
\end{abstract}

Keywords: positron; model potential; wave-packet propagation; vibrational cross-section

\section{Introduction}

A fundamental question in positron-matter interaction is how a positron couples to the vibrational modes of molecules, after experimental measurements [1-3] and theoretical models [4-11] agree appreciably for vibrational excitation cross-sections for low-energy positron-molecule scattering. The observation of this phenomenon in great detail follows the development of high-intensity, monoenergetic positron beams [12,13], which, in turn, cool the source of positron via inelastic collisions, presumably involving vibrational modes [11,14] with a buffer gas of molecules.

The prominent consequence of the strong coupling between the positron and the nuclear degrees of freedom refers to the abnormally large positron annihilation rates observed in polyatomic targets [15-17]. The resonant features of the annihilation spectra have been described using a Breit-Wigner amplitude, which relies on the existence of a positronmolecule bound state or vibrational Feshbach resonance (VFR) [18], mainly populated by the infrared active modes of the molecule $[17,18]$. The VFRs are further enhanced by intramolecular vibrational energy redistribution [18]. On the other hand, the multimodal nature of positron annihilation on molecules was uncovered within a projection operator approach $[19,20]$. This model suggests that resonances in the annihilation phenomenon arise from the formation of a transient positron-molecule complex, with a lifetime long enough to give rise to narrow vibrational resonances via constructive interference $[19,20]$. This model attributes an important role to the correlation-polarization forces, as the mechanism that triggers the positron-molecule attachment, not only involving bound states but virtual states as well $[19,20]$. The coupling of the positron to molecular vibrations appeared to be more intriguing after experimental measurements of annihilation rates revealed that the positron can couple directly to a quasi-continuum of multimode vibrational states [21]. In order to describe the observed data, it was necessary to average the annihilation parameter over all the energetically allowed multimode vibrational excitations, also assuming that the positron couples to the quasi-continuum of states with the same strength [21]. 
The richness of the positron-molecule physics is closely related to the ability of the molecule to bind a positron. As the positron is a light positive particle, the formation of a positron-molecule complex results from the compromise between a flexible attractive electron cloud and a quasi-rigid repulsive nuclei structure. This picture is supported by a collection of experimental [22,23] and theoretical results [24-28] that indicate an strong correlation between positron-molecule binding energies and molecular properties such as dipole moment, polarizability, ionization potential, and number of $\pi$-electrons. However, for a quantitative description of the bonding between a molecule and a positron, a subtle effect should be taken into account, e.g., the nonzero probability amplitude that a target electron tunnels to the positron-attractive well. This effect, which appears as a virtual positronium formation in the many-body positron-molecule self energy, is responsible for a dramatic increase in the positron-molecule attachment [29].

In this work, the interaction of a positron with a diatomic molecule is studied using a potential energy surface (PES) that couples a correlation-polarization potential curve, for the positron-target interaction, with a hydrogenlike harmonic oscillator. On this PES, a time-dependent wave-packet propagation is performed and the cross-section for the excitation of the first vibrational mode of the oscillator is computed, a procedure somewhat similar to that of the reference [30]. In seeking comparison, the mass and natural frequency of the harmonic oscillator were chosen with values close to those known for a hydrogen molecule. The coupling between the molecular vibrational and positron translational modes was described using a linear representation of the $\mathrm{H}_{2}$ polarizabilities as a function of the $\mathrm{H}-\mathrm{H}$ internuclear separation, reported by Kołos and Wolniewicsz [31]. The computed $0 \rightarrow 1$ vibrational excitation cross-section then displays good agreement with previously reported experimental and theoretical results, suggesting that the oscillator embedded in the positron continuum couples to it through a correlation polarization force, which depends on the target oscillatory coordinate. Since the present model employs harmonic approximation, only the $0 \rightarrow 1$ vibrational excitation is accounted for. While more sophisticated positron-vibration couplings could in principle be considered, the available experimental data for $\mathrm{H}_{2}$ are limited to the fundamental vibrational transition.

In the next section, the model potential is described in detail. The wave-packet propagation method is presented in Section 3. The results and discussion are given in Section 4 and some conclusions given in Section 5. Atomic units are assumed throughout the text, unless otherwise stated.

\section{The Model Potential}

The model potential is written as the sum of oscillator (OSC) and positron (POS) components as follows:

$$
V(r, R)=V_{\text {osc }}(R)+V_{\text {pos }}(r, R)
$$

where $r$ is the scattering coordinate for the projectile-target distance relative to the center of mass of the system and $R$ is the internal coordinate of the target vibrational mode.

The OSC term in the above equation is the potential energy of a harmonic oscillator

$$
V_{\text {osc }}(R)=\frac{1}{2} \mu \omega^{2} R^{2}
$$

where the reduced mass is set to $\mu=1000$, corresponding to a homonuclear diatom with atomic mass $M=2000$. The natural frequency $\omega=0.02\left(4389.5 \mathrm{~cm}^{-1}\right)$ provides a harmonic oscillator with parameters close to those of the hydrogen molecule.

The POS term includes the static $\left(V_{s t}\right)$ and the correlation-polarization $\left(V_{c p}\right)$ potentials. The former is represented in the form

$$
V_{s t}(r)=\left(\frac{a_{1}}{r}\right)^{a_{2}} \exp \left(-a_{3} r^{a_{4}}\right)
$$


which reproduces the spherically symmetric static potential energy of $\mathrm{H}_{2}$, at the HarteeFock level, where $\left\{a_{i}\right\}=\{1.1973,2.6633,0.6179,1.2003\}$.

The $V_{c p}$ term, in turn, is written as

$$
V_{c p}(r, R)=-\frac{\alpha(R)}{2 r^{4}} f_{\rho}(r)
$$

where

$$
\alpha(R)=a R+\alpha_{0}
$$

with $a=4.35$ and $\alpha_{0}=5.18$ is a linear approximation (referred to as $1.4 \mathrm{a}_{0}$ ) to the dipole polarizability for $\mathrm{H}_{2}$ from Kołos and Wolniewitz [31].

In Equation (4),

$$
f_{\rho}(r)=1-\exp \left(-\frac{r^{6}}{\rho^{6}}\right)
$$

is the cut-off function proposed by Mitroy and Ivanov [32] in order to damp the $-1 / r^{4}$ term at short distances. The cut-off parameter $\rho$ is chosen to reproduce the desirable result [26,32]. In this case, $\rho=5$ leads to a cross-section close to the experimental values.

Figure 1 shows one-dimensional cuts of the different components of the twodimensional PES in the function of $r$, for $R=0$ and $\rho=5$. The inset shows the ab initio values of the $\mathrm{H}_{2}$ polarizability and the straight line from Equation (5). We observed that with this simple representation, the cross-section was almost indistinguishable from those of a model that fits all the data to a higher degree polynomial. This is so because the average position of the wave-packet in the $R$ coordinate oscillates very close to the origin of the quadratic well.

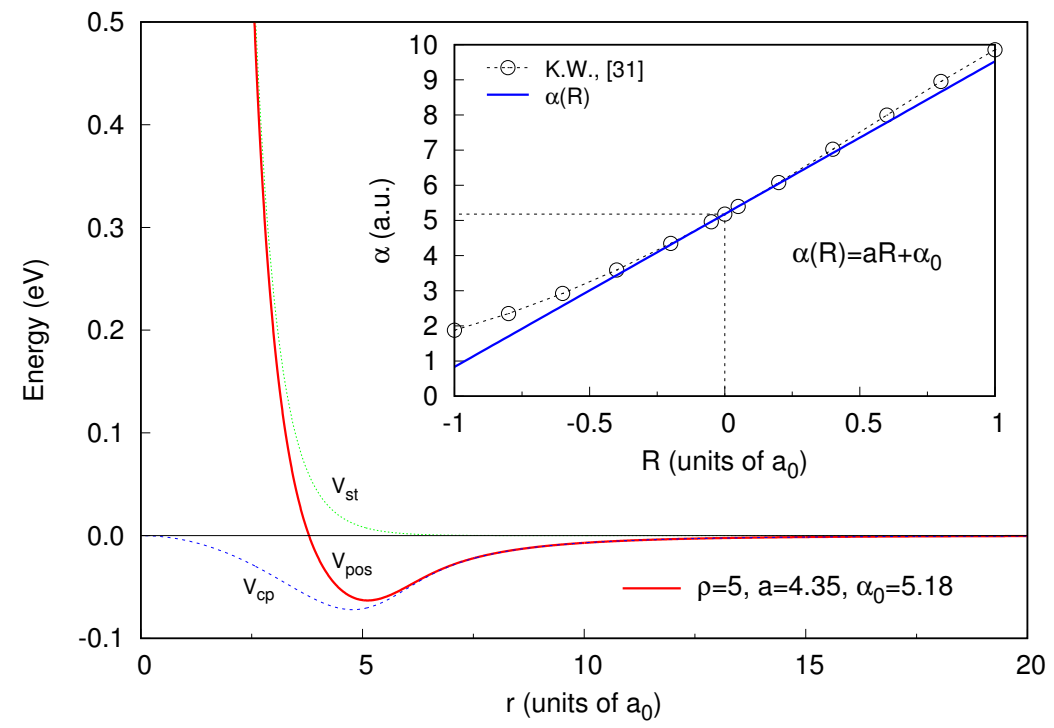

Figure 1. Cuts of the different components of the model potential. The empty dots in the inset are the data from ref. [31]. See text for details.

This model potential assumes a very simple anzatz for the coupling of a low-energy positron to a harmonic vibrational mode. The coupling is included as a parametric dependence of the positron-target correlation-polarization potential $V_{c p}$ on the oscillator coordinate $R$. Note that, here, the target potential remains unchanged under the action of the positron field, a plausible assumption for a small, weakly polarizable molecule as $\mathrm{H}_{2}$. 


\section{Wave-Packet Dynamics}

The time propagation of the wave packet (WP) was performed using a split evolution operator in the form

$$
\Psi(r, R, t+\Delta t)=e^{-i K \frac{\Delta t}{2}} e^{-i V \Delta t} e^{-i K \frac{\Delta t}{2}} \Psi(r, R, t)
$$

where $V$ is the PES from Equation (1) and

$$
K=\frac{p^{2}}{2 m}+\frac{P^{2}}{2 \mu}
$$

is the total kinetic energy operators involving the momenta $p$ and $P$-conjugates of $r$ and $R$, respectively.

In Equation (8), $m$ is the reduced mass of the system defined as

$$
m=\frac{m_{p} M}{m_{p}+M}
$$

where $M=2000$ is the total mass of the oscillator and $m_{p}=1$ is the positron mass.

The initial WP was prepared as the normalized product

$$
\Psi_{i}(r, R, t=0)=g(r) \chi_{i}(R)
$$

where $\chi_{i}(R)$ is the wave function of the $i$-th state of a harmonic oscillator with energy $\varepsilon_{i}$. In the present study, the oscillator is initially in its ground state, $i=0$.

In Equation (10), $g(r)$ represents an incoming Gaussian wave packet

$$
g(r)=\left(\frac{1}{2 \pi \delta r_{0}^{2}}\right)^{1 / 4} e^{-\left(r-r_{0}\right)^{2} / 4 \delta r_{0}^{2}} e^{i k_{0} r}
$$

with initial average position $r_{0}$, initial average momentum $k_{0}$ toward the interaction region, and full width at half maximum (FWHM) $\Delta r_{0}=2 \sqrt{\ln 2} \delta r_{0}$.

Equations (7)-(11) were represented in a discretized grid of the $r \times R$ space with $N_{r} \times N_{R}$ points. At every instant, the WP was propagated using Equation (7) by doing a sequence of forward-backward-forward fast Fourier transforms (FFTs) between the coordinate and momentum spaces.

The cross-section (CS) for the vibrational excitation from state $i$ to state $j$ is obtained by applying a method commonly used for reactive and nonreactive scattering $[33,34]$. The method consists in expanding the outgoing wave function $\Psi\left(r_{\infty}, R, t\right)$ evaluated at large separations $r=r_{\infty}$ in the eigenstates of the oscillator,

$$
\Psi_{i}\left(r=r_{\infty}, R, t\right)=\sum_{f} C_{i f}(t) \chi_{f}(R)
$$

such that the coefficients of the expansion, computed as

$$
C_{i f}(t)=\int_{R_{\min }}^{R_{\max }} \chi_{f}^{*}(R) \Psi_{i}\left(r_{\infty}, R, t\right) d R
$$

represent the flux of the initial WP into the $f$-th vibrational channel after the scattering event.

Hence, the probability of a transition from the vibrational state $i$ to state $j$ of the oscillator will be proportional to the squared modulus of the Fourier transform of the coefficient $C_{i f}(t)$. The $i \rightarrow f$ vibrational excitation CS as a function of the initial positron momentum $k$ is computed as

$$
\sigma_{i f}(k)=\frac{\pi}{m} \frac{k_{f}}{k_{i}}\left|\frac{\tilde{C}_{i f}(E)}{\tilde{g}(k)}\right|^{2}
$$


where $\tilde{C}_{i f}(E)$ and $\tilde{g}(k)$ are the FFTs of Equations (11) and (13), respectively. In Equation (14), $k_{i(j)}=\sqrt{\left.2 m\left[E-\varepsilon_{i(j)}\right)\right]}$, where $E$ is the total energy, which is conserved during the propagation.

The total propagation time was 131,072 atomic units (3.17 ps), large enough for $|C(t)|^{2}$ to fall below $10^{-7}$, thus guaranteeing that the initial WP is completely dissociated. At this point, a problem arises if the WP is partly reflected back into the interaction region at the edges of the grid. Considering that it is impractical to prevent the WP from reaching the grid's edge during the whole time propagation, by using sufficiently large grids, the usual solutions involve the use of a complex absorbing potential in the dissociation regions [35]. In the present case, we choose a more straightforward solution [36], which consists in multiplying the WP, in the last $\Delta r_{\text {damp }}$ dissociation region of the grid, by a damping function

$$
\exp \left(-b_{\text {damp }} \Delta r_{\text {damp }}^{3}\right)
$$

which smoothly decreases from 1 to zero near the grid edge. Due to the quadratic increase in the potential with the $R$ coordinate, it was not necessary to apply a similar damping along the $R$-edge of the rectangular grid. Note further, that the damping should be applied at time intervals $\Delta t_{\text {damp }}$ larger than the propagation time step to avoid nonphysical reflections of the WP.

Table 1 collects the parameters employed in the propagation. The center of the WP was initially placed at $r_{0}=100$ a.u., with an initial kinetic energy of $0.82 \mathrm{eV}$. To ensure that only the outgoing WP is involved in the computation of the coefficient from Equation (13), $r_{\infty}$ was set to 200 a.u. This point is twice as far as the initial WP, which has an initial FWHM of 20 a.u. After going through $r_{\infty}$, the WP was damped with Equation (15) over the region $\Delta r_{\text {damp }}$, which represents almost $80 \%$ of the total size of the grid along the $r$ coordinate. Such a large damping region was necessary to counteract the rapid spreading of the WP, due to the small mass of the projectile. In turn, the values for $\Delta t_{d a m p}$ and $b_{\text {damp }}$ were chosen by trial-and-error, until it was verified that a negligible amount of the dissociated WP reached the edge of the grid.

Table 1. Parameters of the dynamics (in a.u.).

\begin{tabular}{ccc}
\hline Parameter & Value \\
\hline$r_{\text {min }}$ & Grid parameters & \\
$r_{\max }$ & & 0.0 \\
$N_{r}$ & 1200 \\
$R_{\min }$ & 1024 \\
$R_{\max }$ & -1.0 \\
$N_{R}$ & 1.0 \\
$r_{\infty}$ & 32 \\
$\Delta t$ & 200 \\
$\Delta r_{\text {damp }}$ & 0.01 \\
$\Delta t_{\text {damp }}$ & & 950 \\
$b_{\text {damp }}$ & Initial WP parameters & 1000 \\
& & $10^{-7}$ \\
$r_{0}$ & & 100 \\
$\Delta r_{0}$ & & 20 \\
$k_{0}$ & & -0.245 \\
$\Delta k_{0}$ & & 0.14 \\
\hline
\end{tabular}

Note that we used a FFT power-of-two algorithm, implying that the number of grid points must be a power of two along the $r$ and $R$ coordinates. Hence, considering that the the cost of a propagation is very sensitive to the grid size, we first performed a convergence test. Thus, we found that for $N_{r}=2^{10}$ and $N_{R}=2^{5}$, the computed cross- 
section was indistinguishable from those obtained if the grid size was twice as large for either coordinate. Further, as a simple test, it was verified that by representing the quadratic well of Equation (2) with a grid of 32 points in the interval from -1 to 1 , the energy spectra of the oscillator was accurately reproduced by applying a screening technique on the time-dependent propagation [37].

The initial WP was chosen narrow enough in position so that an energy interval wide enough around $0.54 \mathrm{eV}$, the threshold for the first vibrational mode of the oscillator, is well covered. However, note that the narrower the WP in position, the faster it will spread; then, a good compromise was found by setting the FWHM $\Delta r_{0}=20$ a.u. The bandwidth in energy of the initial WP can be estimated as

$$
\Delta E=\frac{\left(k_{0}+\Delta k_{0} / 2\right)^{2}}{2 m}-\frac{\left(k_{0}-\Delta k_{0} / 2\right)^{2}}{2 m}
$$

where $\Delta k_{0}$ is the FWHM of the initial WP in the momentum space.

From Equation (16), $\Delta E \approx 1 \mathrm{eV}$, such that around the average initial incident energy $k_{0}^{2} / 2 m=0.82 \mathrm{eV}$, the cross-section can be reliably described. This can be seen in the red curve of Figure 2, which represents the $0 \rightarrow 1$ vibrational excitation CS, computed with the present method. Notice that the curve is smooth between the threshold energy and $2 \mathrm{eV}$, after which it begins to show a slightly oscillatory behavior, increasing in amplitude with energy. For a good description of the CS for energies above $2 \mathrm{eV}$, The WP should have a FWHM less than 20 a.u., but it would be necessary to choose a new set of damping parameters in order to remove WP reflections at the edge of the grid.

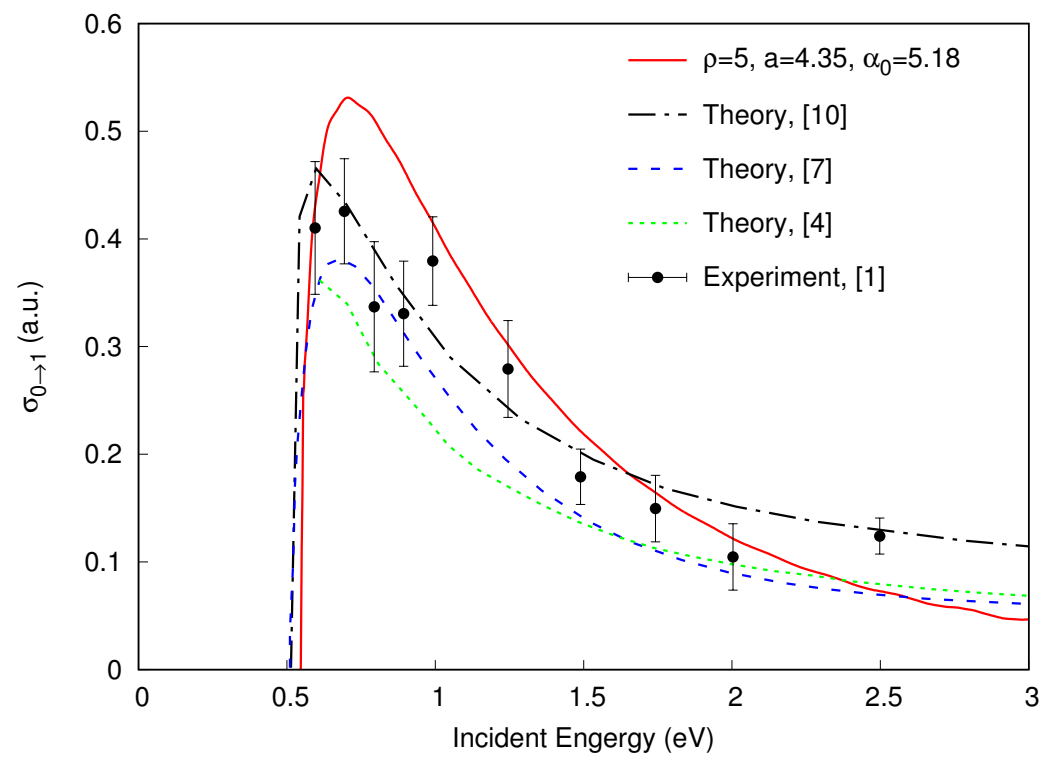

Figure 2. Comparison of the $0 \rightarrow 1$ vibrational excitation CSs, in function of the incident positron energy. The solid red line is the present result.

\section{Results and Discussion}

Figure 2 shows a comparison of the presently computed $0 \rightarrow 1$ vibrational excitation $\mathrm{CS}$, as a function of the initial positron energy, with previous results obtained with other methods [4-6,10]. Our results show the typical behavior of the cross-section with a sharp onset at threshold, followed by a peak with maximum around 0.5 a.u. height, falling down to zero as the energy increases. The good agreement with reported theories $[4-6,10]$ and one experiment [1] suggests that the present model potential is appropriate to describe the $0 \rightarrow 1$ excitation CS of the oscillator. In this sense, it becomes arguable that the coupling between the positron and the first vibrational mode of the target can be accounted for by the instantaneous response of the molecule to the positron through the $R$-dependent attractive correlation polarization potential. 
Figure 3 shows the $0 \rightarrow 1$ vibrational excitation CSs from different PESs obtained by changing, one at a time, the values of the cut-off parameter $\rho$ and the slope $a$ of the $\alpha(R)$ function, given by Equation (5). From the figure, it is clear that a decrease (increase) in the value of $a$ leads to a decrease (increase) in the cross-section as a whole, as expected from the fact that $a$ is a measure of the coupling strength between the translational and vibrational degrees of freedom. In turn, an increase (decrease) of $\rho$ around 5 gives rise to an decrease (increase) in the cross-section, leaving its characteristic shape unchanged.

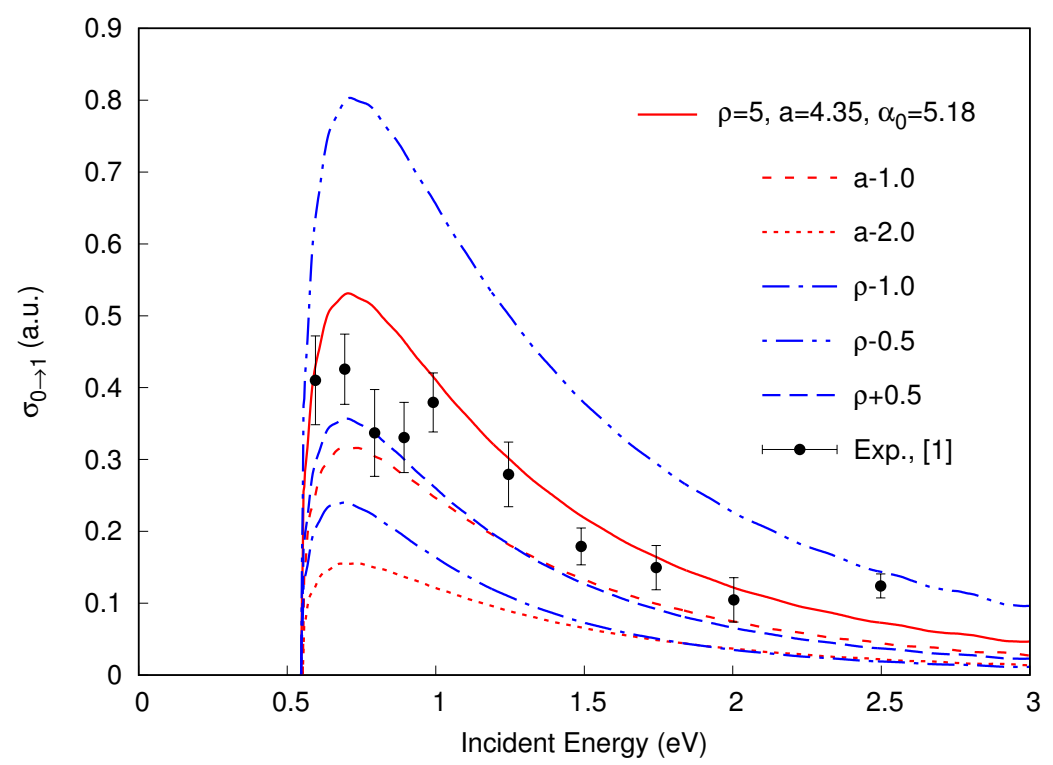

Figure 3. Vibrational excitation CSs in function of the incident energy for different values of the $\rho$ and $a$ parameters in Equations (5) and (6), respectively.

The influence of the parameter $\alpha_{0}$ on the cross-section was also investigated. Figure 4 shows cuts of the PES along the $r$ coordinate for $R=0$, with increasing values of the target polarizability at equilibrium, $\alpha_{0}$. The other parameters of the $V_{c p}$ terms, $\rho$ and $a$, were fixed at 5 and 4.35 , respectively. This trend describes the situation for a positron, which couples with the same strength ( $a=$ const.) to an increasingly polarizable target. The deepest well corresponds to a target eight times more polarizable than that of the hydrogen molecule. Using this family of PESs, propagations were carried out with the same parameters of Table 1 . The resulting $0 \rightarrow 1$ vibrational excitation CSs are shown in Figure 5 . From the figure, it is clear that the cross-section tends to depict a threshold resonance as the target polarizability increases, presumably due to the emergence of a bound state as the potential well becomes deeper.

The above discussion suggests that for the hydrogen molecule, the typical behavior of the $0 \rightarrow 1$ vibrational excitation CS arises from a weak coupling between the positron and the molecular vibration, mainly dominated by the dependence of the positron-target correlation-polarization potential on the vibrational coordinate. In the present model, this is represented by the dependence of the polarizability on the $R$ coordinate. Moreover, as the target becomes more polarizable, the coupling between the positron and the vibrational modes may be enhanced by the emergence of resonances or even bound states. In such a scenario, commonly observed in large polyatomics, the nucleus skeleton of the molecule would be appreciably perturbed by the presence of the positron field; thus, increasing the coupling between the positron and the molecular vibration. 


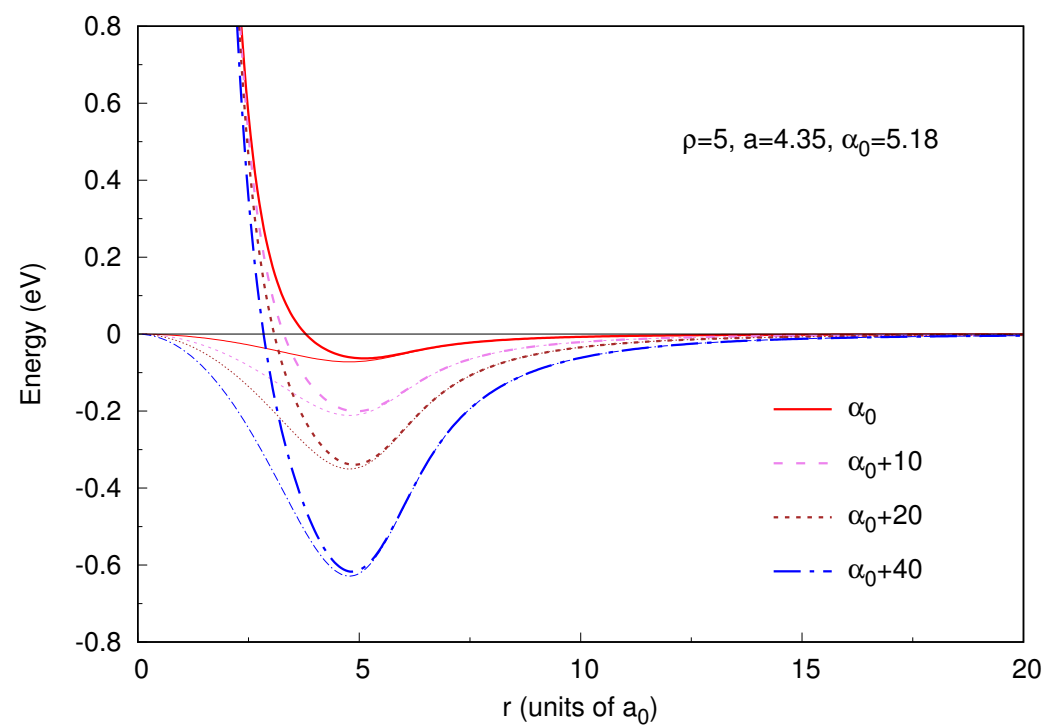

Figure 4. Cuts of the PES for increasing values of $\alpha_{0}$.

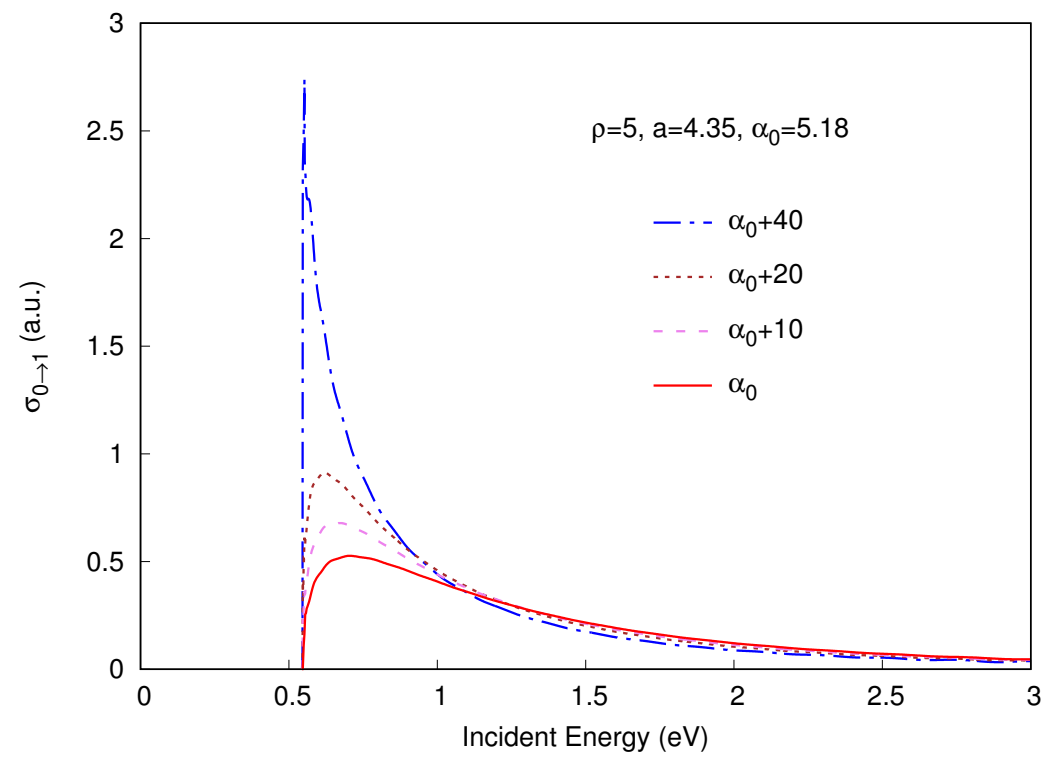

Figure 5. $0 \rightarrow 1$ vibrational excitation CSs obtained with the different PESs from the Figure 4 .

\section{Conclusions}

In this work, we show that a simple model that couples a harmonic oscillator to a positron through a correlation-polarization potential can capture the main physics involved in a single-mode vibrational excitation by positron impact. Here, this phenomenon was studied using a time-dependent wave-packet propagation. The $0 \rightarrow 1$ vibrational excitation cross-section was computed by projecting, at every time step, the dissociated wave-packet with the first excited state of the oscillator. The cross-section shows good agreement with reported values and suggests that the target vibration can be coupled to the positron continuum by the dependence of the target polarizability on the vibrational coordinate. On the other hand, the $0 \rightarrow 1$ vibrational excitation cross-section tends to depict a threshold resonance for an increasingly polarizable target. Future work will be devoted to studying positron coupling with higher vibrational modes, in which case, a more realistic description of the oscillator beyond the harmonic approximation would be required. 
Author Contributions: Conceptualization, L.A.P., M.T.d.N.V. and J.R.M.; Data curation, L.A.P.; Funding acquisition, J.R.M.; Investigation, L.A.P., M.T.d.N.V. and J.R.M.; Methodology, L.A.P. and M.T.d.N.V.; Project administration, J.R.M.; Resources, J.R.M.; Software, M.T.d.N.V.; Supervision, J.R.M.; Writing—original draft, L.A.P.; Writing—review \& editing, L.A.P., M.T.d.N.V. and J.R.M. All authors have read and agreed to the published version of the manuscript.

Funding: This research was funded by Conselho Nacional de Desenvolvimento Científico e Tecnológico grant numbers 304571/2018-0 and 307125/2019-0.

Institutional Review Board Statement: Not applicable.

Informed Consent Statement: Not applicable.

Acknowledgments: Luis Poveda thanks Carlos Magno and Luis d'Fonseca for helpful discussions. Marcio Varella and José Mohallem acknowledge support from the Brazilian agency Conselho Nacional de Desenvolvimento Científico e Tecnológico (CNPq), Grants: 304571/2018-0 and 307125/20190 , respectively.

Conflicts of Interest: The authors declare no conflict of interest.

\section{References}

1. Sullivan, J.P.; Gilbert, S.J.; Surko, C.M. Excitation of Molecular Vibrations by Positron Impact. Phys. Rev. Lett. 2001, 86, 1494. [CrossRef]

2. Gilbert, S.J.; Greaves, R.G.; Surko, C.M. Positron Scattering from Atoms and Molecules at Low Energies. Phys. Rev. Lett. 1999, 82, 5032-5035. [CrossRef]

3. Marler, J.P.; Surko, C.M. Systematic comparison of positron- and electron-impact excitation of the $v_{3}$ vibrational mode of $\mathrm{CF}_{4}$. Phys. Rev. A 2005, 72, 062702. [CrossRef]

4. Gianturco, F.A.; Mukherjee, T. Dynamical coupling effects in the vibrational excitation of $\mathrm{H}_{2}$ and $\mathrm{N}_{2}$ colliding with positrons. Phys. Rev. A 1997, 55, 1044-1055. [CrossRef]

5. Mukherjee, T.; Ghosh, A.S.; Jain, A. Low-energy positron collisions with $\mathrm{H}_{2}$ and $\mathrm{N}_{2}$ molecules by using a parameter-free positron-correlation-polarization potential. Phys. Rev. A 1991, 43, 2538-2543. [CrossRef] [PubMed]

6. Varella, M.T.D.N.; Lima, M.A.P. Near-threshold vibrational excitation of $\mathrm{H}_{2}$ by positron impact: A projection-operator approach Phys. Rev. A 2007, 76, 052701. [CrossRef]

7. Varella, M.T.D.N.; de Oliveira, E.M.; Lima, M.A. Near threshold vibrational excitation of molecules by positron impact: A projection operator approach. Nucl. Instr. Meth. B 2008, 266, 435-440. [CrossRef]

8. Mazon, K.T.; Tenfen, W.; Michelin, S.E.; Arretche, F.; Lee, M.T.; Fujimoto, M.M. Vibrational cross sections for positron scattering by nitrogen molecules. Phys. Rev. A 2010, 82, 032704. [CrossRef]

9. Mukherjee, T.; Mukherjee, M. Low-energy positron-nitrogen-molecule scattering: A rovibrational close-coupling study. Phys. Rev. A 2015, 91, 062706. [CrossRef]

10. Zammit, M.C.; Fursa, D.V.; Savage, J.S.; Bray, I.; Chiari, L.; Zecca, A.; Brunger, M.J. Adiabatic-nuclei calculations of positron scattering from molecular hydrogen. Phys. Rev. A 2017, 95, 022707. [CrossRef]

11. Poveda, L.A.; Assafrão, D.; Pinheiro, J.G.; Mohallem, J.R. Close-coupling scattering cross sections and a model for positron cooling in a buffer gas of molecular nitrogen. Phys. Rev. A 2019, 100, 062706. [CrossRef]

12. Gilbert, S.J.; Kurz, C.; Greaves, R.G.; Surko, C.M. Creation of a monoenergetic pulsed positron beam. App. Phys. Lett. 1997, 70, 1944-1946. [CrossRef]

13. Danielson, J.R.; Dubin, D.H.E.; Greaves, R.G.; Surko, C.M. Plasma and trap-based techniques for science with positrons. Rev. Mod. Phys. 2015, 87, 247-306. [CrossRef]

14. Natisin, M.R.; Danielson, J.R.; Surko, C.M. Positron cooling by vibrational and rotational excitation of molecular gases. J. Phys. B At. Mol. Opt. Phys. 2014, 47, 225209. [CrossRef]

15. Young, J.A.; Surko, C.M. Feshbach-resonance-mediated positron annihilation in small molecules. Phys. Rev. A 2008, 78, 032702. [CrossRef]

16. Young, J.A.; Surko, C.M. Feshbach-resonance-mediated annihilation in positron interactions with large molecules. Phys. Rev. A 2008, 77, 052704. [CrossRef]

17. Gribakin, G.F.; Young, J.A.; Surko, C.M. Positron-molecule interactions: Resonant attachment, annihilation, and bound states. Rev. Mod. Phys. 2010, 82, 2557-2607. [CrossRef]

18. Gribakin, G.F.; Lee, C.M.R. Positron Annihilation in Molecules by Capture into Vibrational Feshbach Resonances of InfraredActive Modes. Phys. Rev. Lett. 2006, 97, 193201. [CrossRef]

19. d'A Sanchez, S.; Lima, M.A.P.; Varella, M.T.D.N. Feshbach projection operator approach to positron annihilation. Phys. Rev. A 2009, 80, 052710. [CrossRef]

20. d'A Sanchez, S.; Lima, M.A.; Varella, M.T.D.N. Multimode vibrational couplings in resonant positron annihilation. Phys. Rev. Lett. 2011, 107, 103201. [CrossRef] 
21. Jones, A.C.L.; Danielson, J.R.; Natisin, M.R.; Surko, C.M.; Gribakin, G.F. Ubiquitous Nature of Multimode Vibrational Resonances in Positron-Molecule Annihilation. Phys. Rev. Lett. 2012, 108, 093201. [CrossRef]

22. Danielson, J.R.; Young, J.A.; Surko, C.M. Analysis of experimental positron-molecule binding energies. J. Phys. Conf. Ser. 2010, 199, 012012. [CrossRef]

23. Danielson, J.R.; Jones, A.C.L.; Gosselin, J.J.; Natisin, M.R.; Surko, C.M. Interplay between permanent dipole moments and polarizability in positron-molecule binding. Phys. Rev. A 2012, 85, 022709. [CrossRef]

24. Amaral, P.H.R.; Mohallem, J.R. Positron binding to atoms and apolar molecules: A convergence of theory and experiment. Phys. Rev. A 2012, 86, 042708. [CrossRef]

25. Romero, J.; Charry, J.A.; Flores-Moreno, R.; Varella, M.T.D.N.; Reyes, A. Calculation of positron binding energies using the generalized any particle propagator theory. J. Chem. Phys. 2014, 141, 114103. [CrossRef] [PubMed]

26. Swann, A.R.; Gribakin, G.F. Positron Binding and Annihilation in Alkane Molecules. Phys. Rev. Lett. 2019, 123, 113402. [CrossRef]

27. Suzuki, H.; Otomo, T.; Iida, R.; Sugiura, Y.; Takayanagi, T.; Tachikawa, M. Positron binding in chloroethenes: Modeling positron-electron correlation-polarization potentials for molecular calculations. Phys. Rev. A 2020, 102, 052830. [CrossRef]

28. Amaral, P.H.R.; Mohallem, J.R. Machine-learning predictions of positron binding to molecules. Phys. Rev. A 2020, 102, 052808 . [CrossRef]

29. Hofierka, J.; Cunningham, B.; Rawlins, C.M.; Patterson, C.H.; Green, D.G. Many-body theory of positron binding in polyatomic molecules. arXiv 2021, arXiv:2105.06959.

30. Sugiura, Y.; Suzuki, K.; Koido, S.; Takayanagi, T.; Kita, Y.; Tachikawa, M. Quantum dynamics calculation of the annihilation spectrum for positron-proline scattering. Comp. Theor. Chem. 2019, 1147, 1-7. [CrossRef]

31. Kołos, W.; Wolniewicz, L. Polarizability of the Hydrogen Molecule. J. Chem. Phys. 1967, 46, 1426-1432. [CrossRef]

32. Mitroy, J.; Ivanov, I.A. Semiempirical model of positron scattering and annihilation. Phys. Rev. A 2002, 65, 042705. [CrossRef]

33. Marston, C.; Balint-Kurti, G.; Dixon, R. Time dependent quantum dynamics of reactive scattering and the calculation of product quantum state distributions-A study of the collinear $\mathrm{F}+\mathrm{H}_{2}(\mathrm{v}=0)->\mathrm{HF}\left(\mathrm{v}^{\prime}\right)+\mathrm{H}$ reaction. Theor. Chim. Acta 1991, 79, 313-322. [CrossRef]

34. Bradley, K.S.; Schatz, G.C.; Balint-Kurti, G.G. Wave Packet Methods for the Direct Calculation of Energy-Transfer Moments in Molecular Collisions. J. Phys. Chem. A 1999, 103, 947-952. [CrossRef]

35. Vibók, Á.; Halász, G.J. Parametrization of complex absorbing potentials for time-dependent quantum dynamics using multi-step potentials. Phys. Chem. Chem. Phys. 2001, 3, 3048-3051. [CrossRef]

36. Dixon, R.N.; Marston, C.C.; Balint-Kurti, G.G. Photodissociation dynamics and emission spectroscopy of $\mathrm{H}_{2} \mathrm{~S}$ in its first absorption band: A time dependent quantum mechanical study. J. Chem. Phys. 1990, 93, 6520-6534. [CrossRef]

37. Takatsuka, K.; Hashimoto, N. A novel method to calculate eigenfunctions and eigenvalues in a given energy range. J. Chem. Phys. 1995, 103, 6057-6067. [CrossRef] 